\title{
SIMULATION OF A HIGH-FREQUENCY LINK OF PHASE COMPARISON PROTECTION OF TRANSMISSION LINES FOR OPTIMIZATION ITS SETTINGS
}

\author{
Nikolay Yu. Ruban ${ }^{1,}$, Almaz O. Sulaymanov ${ }^{1}$, Ruslan A. Ufa ${ }^{1}$, and Igor A. Razzhivin ${ }^{1}$ \\ ${ }^{1}$ Tomsk Polytechnic University, 634050, Tomsk, Russia
}

\begin{abstract}
The objective of this work is to determine the influence of highfrequency link (HFL) on functioning of phase comparison protection (PCP) through the usage of detailed models that take into account specific features of the PCP and processes in HFL. The article describes the case of the most common variant of the high frequency signal transmission by using a scheme 'phase-to-ground'. To verify suggested solution the developed mathematical model of HFL has been studied. Presented simulation results confirm the effectiveness of the proposed approach for solving the problem.
\end{abstract}

\section{Introduction}

One of the main reasons for wrong actions of the phase comparison protection (PCP) is inappropriate setting for the particular implementation and operating conditions. It is related to over-generalized view of the errors generated by measuring transformers and actually by apparatus of the used protection, as well as the use of insufficiently reliable primary regime information [1]. This means that, in accordance with the existing methods for calculating relay protection (RP) settings, accounting the actual conditions of the functioning of the particular implementation of protection is carried out very simplistically - through a variety of generalized adjusting coefficients. The latter is due to the fact that the software systems used to determine the RP settings in which the calculation is made in strict accordance with existing procedures, as well as systems for modelling regimes and processes in electric power systems (EPS) is used several limitations and assumptions. This is connected with a numerical method for solving differential equations [2, 3], and therefore reproducible primary regime information and its transformation by particular implementations of PCP contains errors.

At the same time, the main sources of errors and consequently, the main cause of incorrect PCP settings are its measuring and transmitting parts.

Transmitting part (TP) is a high-frequency component of the protection, and regardless of the type of execution comprises: a part of transmission line (PL), linking half-sets at opposite ends of the protected line; high-frequency chokes including a reactor (R),

*Corresponding author: rubanny@tpu.ru 
equivalent capacitance (E) and inductivity; coupling capacitors (CC); connecting filters (CF) and high-frequency cables (HFC) for each end of the protected line, respectively. The above elements of TP constitute an electric circuit comprising RLC elements that significantly affect the transmitted signal. In addition to the mentioned elements, in its structure also high-frequency signal receivers and transmitters (RT) are included, together they combine a high frequency link (HFL) of PCP.

\section{Mathematical simulation of relay protection}

Thus for PCP modelling is also advisable to take into account the impact on its functioning HFL, the elements of which inevitably have an impact on the transmitted signal.

Figure 1 shows the equivalent circuit of HFL.

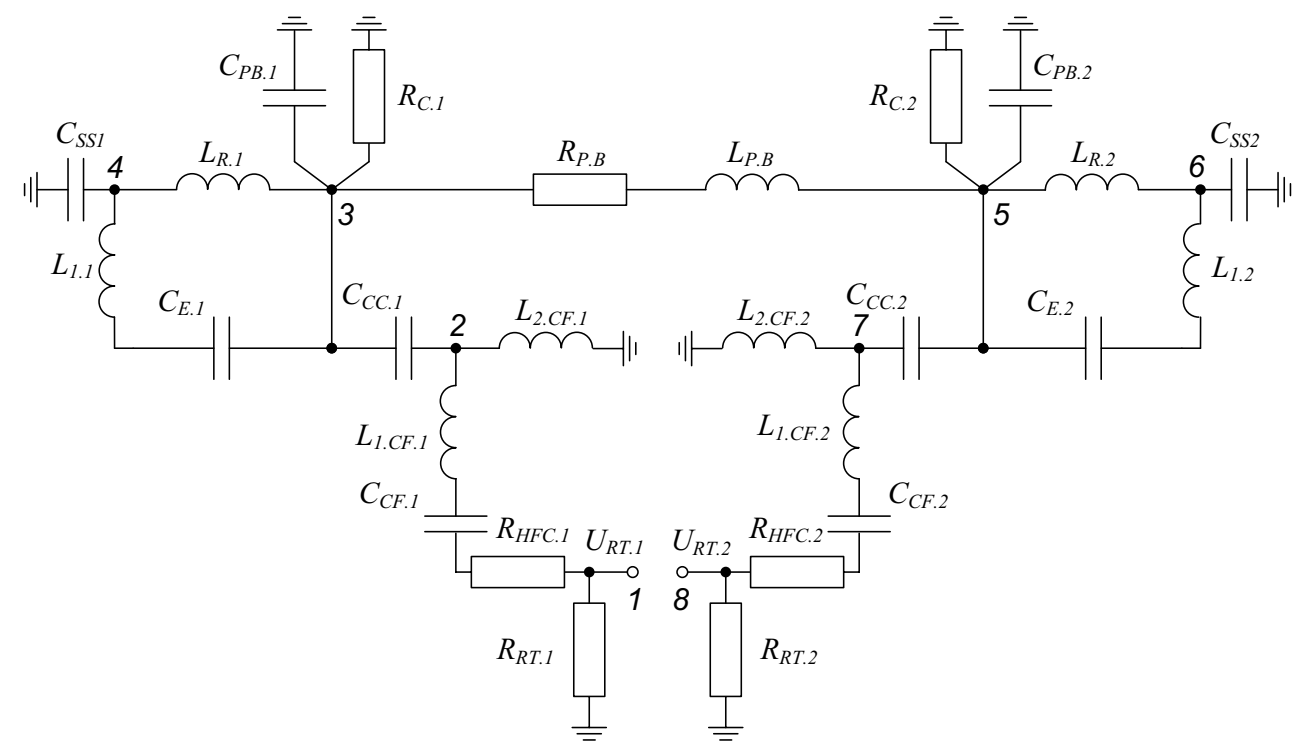

Fig. 1. Equivalent circuit of HFL.

On the basis of the equivalent circuit considering the relationship HFL functional blocks, which is carried out by the determination of appropriate equivalent load resistance of these blocks, is formed the differential equation describing the processes in HFL.

$$
\begin{aligned}
& \frac{d^{15}\left(u_{\text {OUT.HFL1 }}(t) \cdot A_{2 H F L 1}\right)}{d t^{15}}+\frac{d^{14}\left(u_{\text {OUT.HFL1 }}(t) \cdot B_{2 H F L 1}\right)}{d t^{14}}+\frac{d^{13}\left(u_{\text {OUT.HFL }}(t) \cdot C_{2 H F L 1}\right)}{d t^{13}}+ \\
& +\frac{d^{12}\left(u_{\text {OUT.HFLL }}(t) \cdot D_{2 H F L 1}\right)}{d t^{12}}+\frac{d^{11}\left(u_{\text {OUT.HFL1 }}(t) \cdot E_{2 H F L 1}\right)}{d t^{11}}+\frac{d^{10}\left(u_{\text {OUT.HFL1 }}(t) \cdot F_{2 H F L 1}-u_{\text {IN.HFL }}(t) \cdot F_{1 H F L 1}\right)}{d t^{10}}+ \\
& +\frac{d^{9}\left(u_{\text {OUT.HFL1 }}(t) \cdot G_{2 H F L 1}\right)}{d t^{9}}+\frac{d^{8}\left(u_{\text {OUT.HFL1 }}(t) \cdot H_{2 H F L 1}-u_{I N . H F L 1}(t) \cdot H_{1 H F L 1}\right)}{d t^{8}}+\frac{d^{7}\left(u_{\text {OUT.HFL1 }}(t) \cdot I_{2 H F L 1}\right)}{d t^{7}}+ \\
& +\frac{d^{6}\left(u_{\text {OUT.HFL1 }}(t) \cdot J_{2 H F L 1}-u_{I N . H F L 1}(t) \cdot J_{1 H F L 1}\right)}{d t^{6}}+\frac{d^{5}\left(u_{\text {OUT.HFLL }}(t) \cdot K_{2 H F L 1}\right)}{d t^{5}}+\frac{d^{4}\left(u_{\text {OUT.HFL1 }}(t) \cdot L_{2 H F L 1}\right)}{d t^{4}}+ \\
& +\frac{d^{3}\left(u_{\text {OUT.HFL1 }}(t) \cdot M_{2 H F L 1}\right)}{d t^{3}}+\frac{d^{2}\left(u_{\text {OUT.HFLL }}(t) \cdot N_{2 H F L 1}\right)}{d t^{2}}+\frac{d\left(u_{\text {OUT.HFL1 }}(t) \cdot O_{2 H F L 1}\right)}{d t}+u_{\text {OUT.HFL1 }}(t) \cdot P_{2 H F L 1}=0
\end{aligned}
$$

Factors $F_{1 H F L 1}, H_{1 H F L 1}, J_{1 H F L 1}$ and $A_{2 H F L 1}-P_{2 H F L 1}$ are presented in general form and are not given in the article because of the large size. 


\section{Experimental results}

As a preliminary tests of studies with a programs MathCAD and MATLAB Simulink are determined amplitude-frequency and phase-frequency characteristics of the HFL mathematical model necessary to assess the amplitude and phase errors for the particular frequency of the converted signal. They are shown in Figure 2.

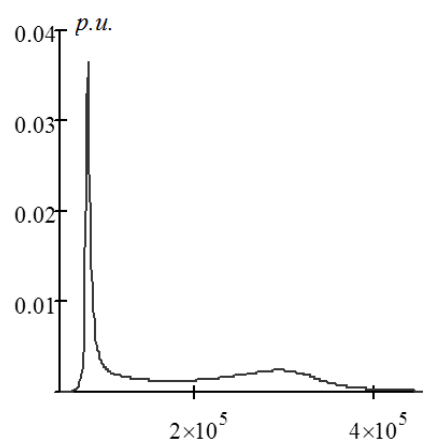

a)

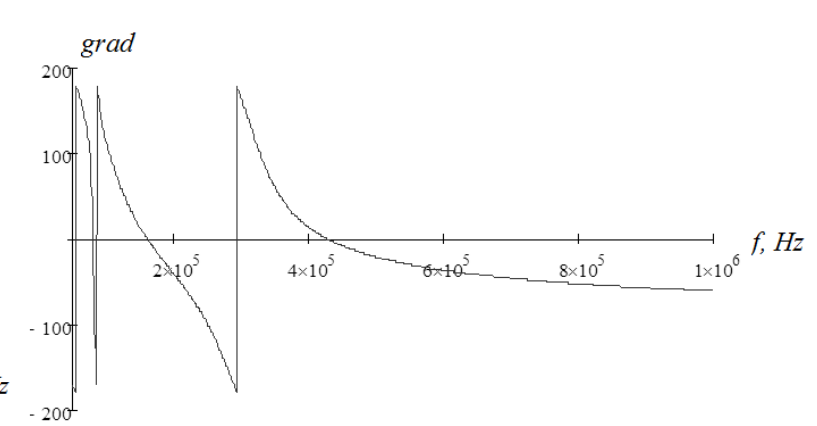

b)

Fig. 2. Amplitude-frequency (a) and phase-frequency (b) characteristics of the HFL mathematical model.

After the pre-tests studies obtained mathematical model is converted into a program code and integrated into the specialized program for PCP simulation - DPPmodels $[4,5]$, previously developed by the author. The program allows to set the parameters of HFL and with the integrated oscilloscope to analyze the impact of changes in these parameters on the transmitted signal.

For example for double ended lines setpoint of PCP blocking angle should be selected as short as possible considering signal propagation delay and the necessary reserve. The value of reserve on the blocking angle is recommended to choose a minimum of $30^{\circ}$ using the same half-sets of PCP. The propagation delay of the signal is assumed to be $6^{\circ}$ for every $100 \mathrm{~km}$ of line, that is typical for HFL signal transmission. Often, however, this value is chosen significantly enhanced. Using the developed tool for PCP modeling, including HFT, allows to carry out a substantiated calculation of reserve angle. Example oscillograms confirming features of the program DPPmodels are shown in Figure 3.

The oscillograms presents HF signals at the transmitter output and the receiver input, logical signals at the input and output of the phase comparison element of the first half-set of PCP. 


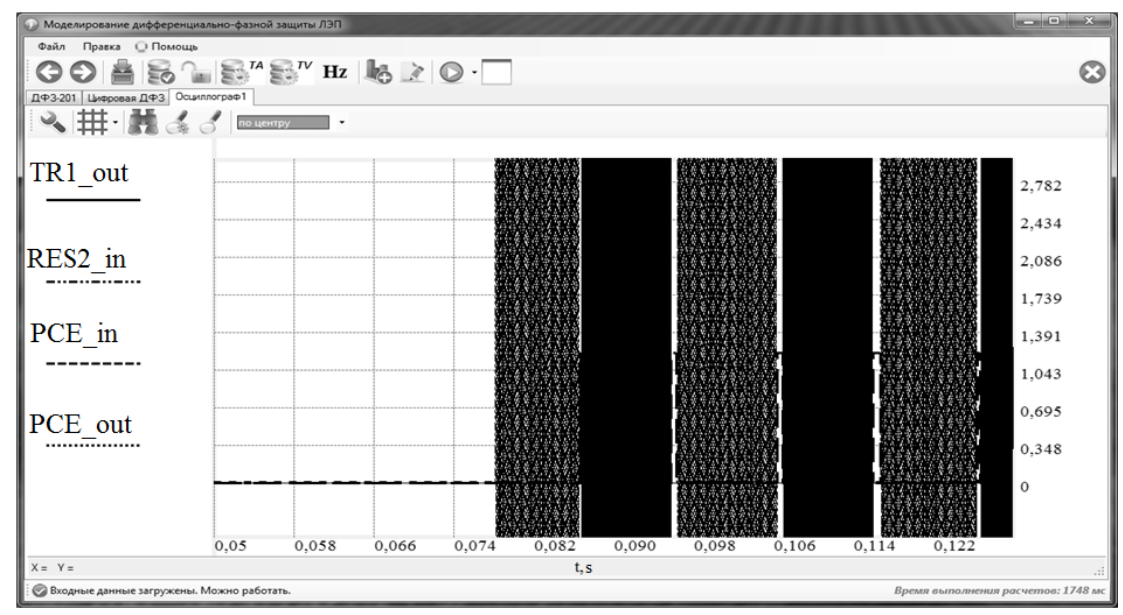

Fig. 3. Oscillograms of HF and logical signals.

Oscillograms confirm the ability to accurately determine the offset between the HF signals, limited by value of blocking angle.

\section{Conclusions}

The synthesized model provides adequate reproduction of processes in a high-frequency part of the PCP. Thus it is achieved the possibility of determining and accounting of the actual errors of the HFL elements formed by a particular spectrum of input regime values. At the same time errors definition of the transmission signal through the HFL becomes particularly urgent in connection with the tendency to increase the use of FACTS in Russian networks with different capacitive filter-compensating devices, connection of which has an impact on transient processes [6]. The results confirmed the possibility of using the proposed approach for the determination of errors in a HFL.

The work was supported by the Ministry of Education and Science of the Russian Federation under the governmental grant "Science" №3901 (Research and development of the hybrid model of the back-to-back high-voltage direct current transmission system).

\section{References}

1. M. Andreev, A. Sulaymanov, A. Gusev, 13th IET International Conference on Developments in Power System Protection, 539 (2016)

2. G. Dahlquist, Math. Scand., 4, 33 (1956)

3. J. D. Lambert, Computational methods in ordinary differential equations (John Wiley and Sons, Ltd., New Jersey, 1973)

4. N. Ruban, A. Gusev, A. Pishchulin, MATEC Web Conf., 37, 01046 (2015)

5. A. Suvorov, Y. Borovikov, A. Gusev, A. Sulaymanov, M. Andreev, N. Ruban, R. Ufa, Electrical Engineering (to be published)

6. L. Balyberdin, E. Zmaznov, Yu. Kraichik, B. Krasnova, N. Lozinova, M. Mazurov, G. Prochan, Power Technology and Engineering, 38, 119 (2004) 